\title{
Synthesis of Enantiopure Fluorohydrins Using Alcohol Dehydrogenases at High Substrate Concentrations
}

\author{
Wioleta Borzęcka, Iván Lavandera, * and Vicente Gotor*
}

Departamento de Química Orgánica e Inorgánica, Instituto Universitario de Biotecnología de Asturias, University of Oviedo, C/Julián Clavería 8, 33006 Oviedo, Spain

Tel.: +34 985103452 or +34 985 103448; Fax: +34 985 103448; E-mail: lavanderaivan@uniovi.es or vgs@uniovi.es.

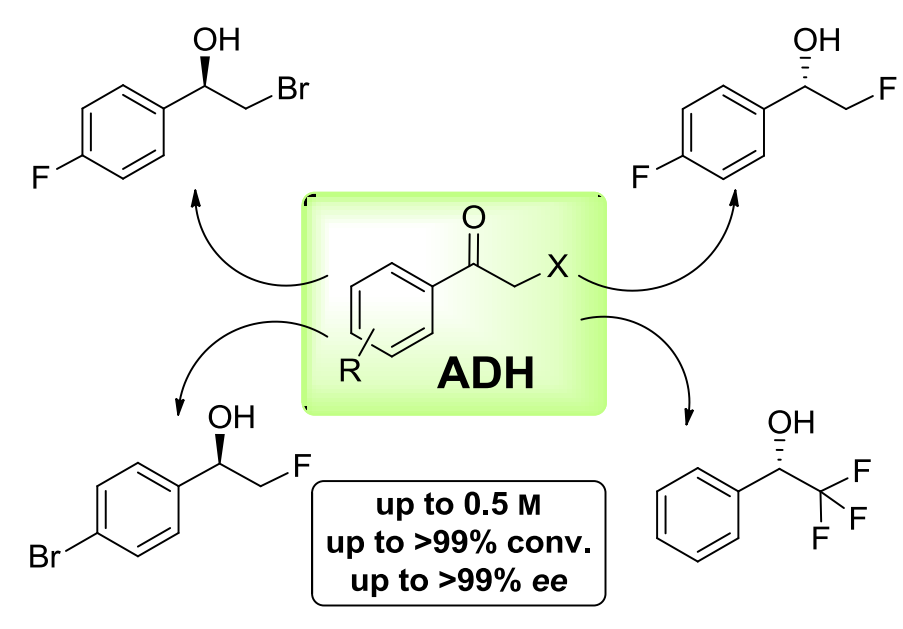

Abstract. The use of purified and overexpressed alcohol dehydrogenases to synthesize enantiopure fluorinated alcohols is shown. Performing the bioreductions with ADH-A from Rhodococcus ruber overexpressed in E. coli, no external cofactor was necessary to obtain the enantiopure (R)-derivatives. Employing Lactobacillus brevis ADH, it was possible to achieve the synthesis of enantiopure (S)fluorohydrins at $0.5 \mathrm{M}$ substrate concentration. Furthermore, due to the activated character of these substrates, it was not necessary a huge excess of the hydrogen donor. 
Fluorinated compounds have a central role nowadays in pharmaceuticals, agrochemicals, radiotracers, and high performance materials. ${ }^{1}$ Particularly, vicinal fluoro alcohols (fluorohydrins), also present interesting properties as liquid crystals ${ }^{2}$ and as precursors of natural product analogues such as steroids or carbohydrates, ${ }^{3}$ since it is well-known that the introduction of $\mathrm{C}-\mathrm{F}$ bonds can modulate their physicochemical properties, including bioavailability, lipophilicity, and oxidative stability.

To obtain these derivatives in a selective fashion, recently several strategies have been described ${ }^{4}$ and among them, the aperture of racemic or meso-epoxides, ${ }^{5}$ the $\mathrm{C}-\mathrm{C}$ formation of (partially) fluorinated building blocks ${ }^{6}$ or the reduction of $\alpha$-fluoro ketones ${ }^{7}$ can be highlighted. Although remarkable improvements have been done in these fields in the past years, the use of enzymes ${ }^{8}$ to synthesize chiral alcohols is very competitive in terms of selectivity and environmentally friendly conditions. Thus, in the particular case of the bioreduction of fluorinated ketone precursors, historically whole cells were employed to obtain enantioenriched fluorohydrins, ${ }^{9}$ but due to the presence of several enzymes with opposite stereopreference, in many examples it was not possible to obtain the enantiopure alcohols. Therefore, in the last few years the use of isolated or overexpressed alcohol dehydrogenases (ADHs) ${ }^{10}$ in combination with very efficient nicotinamide recycling systems has been successfully shown to synthesize fluorinated alcohols in excellent yields and $e{ }^{11}$

Most of the biocatalytic examples achieved until now are related with the reduction of 2fluoroacetophenone and 2,2,2-trifluoroacetophenone, 9,11 so due to the relevance of chiral aromatic fluorohydrins, ${ }^{2,12}$ we became interested in achieving a systematic study about the application of purified and overexpressed ADHs with opposite stereopreference to obtain both enantiopure antipodes of the fluorohydrin derivatives.

A series of $\alpha$-fluoroacetophenones (3b-l) were synthesized through bromination of the corresponding acetophenones 1 with $N$-bromosuccinimide (NBS) and $p$-toluenesulfonic acid ( $p$-TsOH) in acetonitrile $(\mathrm{MeCN})$ at $50^{\circ} \mathrm{C}$ to obtain the $\alpha$-bromo ketones $2,{ }^{13}$ followed by nucleophilic substitution using $\mathrm{KF}$ and $\mathrm{ZnF}_{2}$ in $\mathrm{MeCN}$ at $100^{\circ} \mathrm{C}$ (Scheme 1). ${ }^{14}$ 2-Fluoroacetophenone (3a) was prepared as previously 
described. ${ }^{15}$ The synthesis of the corresponding racemic fluorohydrins was performed by simple treatment of the ketones with $\mathrm{NaBH}_{4}$ in $\mathrm{MeOH}$ at $0^{\circ} \mathrm{C}$.

Scheme 1. Synthesis of $\alpha$-fluoroacetophenone derivatives 3a-l

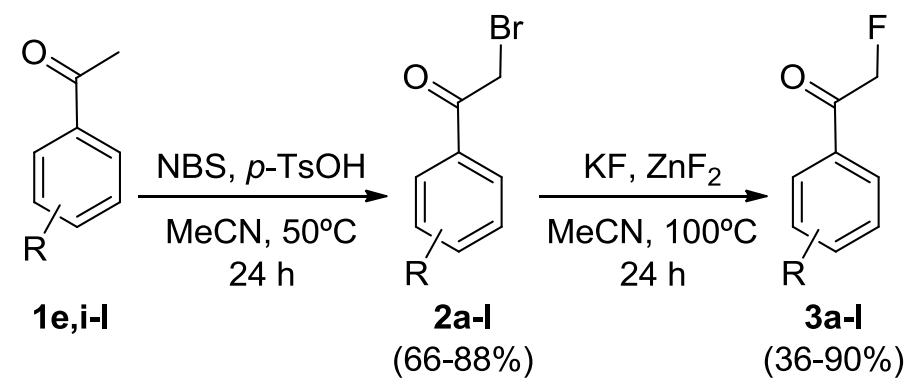

a, $\mathrm{R}=\mathrm{H} ; \mathbf{b}, \mathrm{R}=p-\mathrm{F} ; \mathbf{c}, \mathrm{R}=p-\mathrm{Cl} ; \mathbf{d}, \mathrm{R}=p-\mathrm{Br}$

$\mathbf{e}, \mathrm{R}=p-\mathrm{l} ; \mathbf{f}, \mathrm{R}=p-\mathrm{Me} ; \mathbf{g}, \mathrm{R}=p-\mathrm{OMe} ; \mathbf{h}, \mathrm{R}=p-\mathrm{NO}_{2}$

$\mathbf{i}, \mathrm{R}=m-\mathrm{F} ; \mathbf{j}, \mathrm{R}=m-\mathrm{Cl} ; \mathbf{k}, \mathrm{R}=m-\mathrm{OMe} ; \mathrm{I}, \mathrm{R}=m-\mathrm{NO}_{2}$

Due to our previous expertise in ADH-catalyzed reductions, ${ }^{13,16}$ we selected a series of purified and overexpressed biocatalysts (see the Experimental Section), to achieve the transformations using ketones 3a-l as substrates, together with fluorinated $\alpha$-bromoacetophenones $\mathbf{2 b}$ and $\mathbf{2 i}$, and also 2,2-difluoro- (4) and 2,2,2-trifluoroacetophenone (5) to compare the activity and selectivity with the monofluorinated compound 3a. Buffer Tris. $\mathrm{HCl} 50 \mathrm{mM}$ pH 7.5 was selected as appropriate medium, except for brominated compounds $\mathbf{2} \mathbf{b}$ and $\mathbf{2} \mathbf{i}$ where Tris. $\mathrm{H}_{2} \mathrm{SO}_{4}$ was employed to avoid undesired $\mathrm{S}_{\mathrm{N}} 2$ reactions. ${ }^{13}$ Furthermore, $1 \mathrm{mM}$ of the corresponding nicotinamide cofactor was also added and 2-propanol or glucose with glucose dehydrogenase $(\mathrm{GDH})$ to recycle it. After enzymatic screening, overexpressed ADH-A from Rhodococcus ruber in E. coli (E. coli/ADH-A), ${ }^{17}$ and commercially available ADH from Lactobacillus brevis (LBADH) ${ }^{18}$ were chosen as the best biocatalysts in terms of activity and selectivity. The employment of these enzymes is highly desirable since they can work under the 'coupled-substrate' approach using 2-propanol to recycle the cofactor, diminishing processes costs and allowing higher substrate concentrations due to better ketone solubility in the aqueous buffer. Furthermore, due to the opposite stereopreference they show, it was possible to get access to both alcohol enantiomers. 
Therefore, substrates $\mathbf{2 b}, \mathbf{2 i}, \mathbf{3 a}-\mathbf{l}, \mathbf{4}$ and $\mathbf{5}(30 \mathrm{mM})$ were subjected under these biocatalytic conditions obtaining excellent results with both enzymes (Table 1).

Table 1. Bioreductions of fluorinated ketones (30 mM) using E. coli/ADH-A and $\mathrm{LBADH}^{a}$

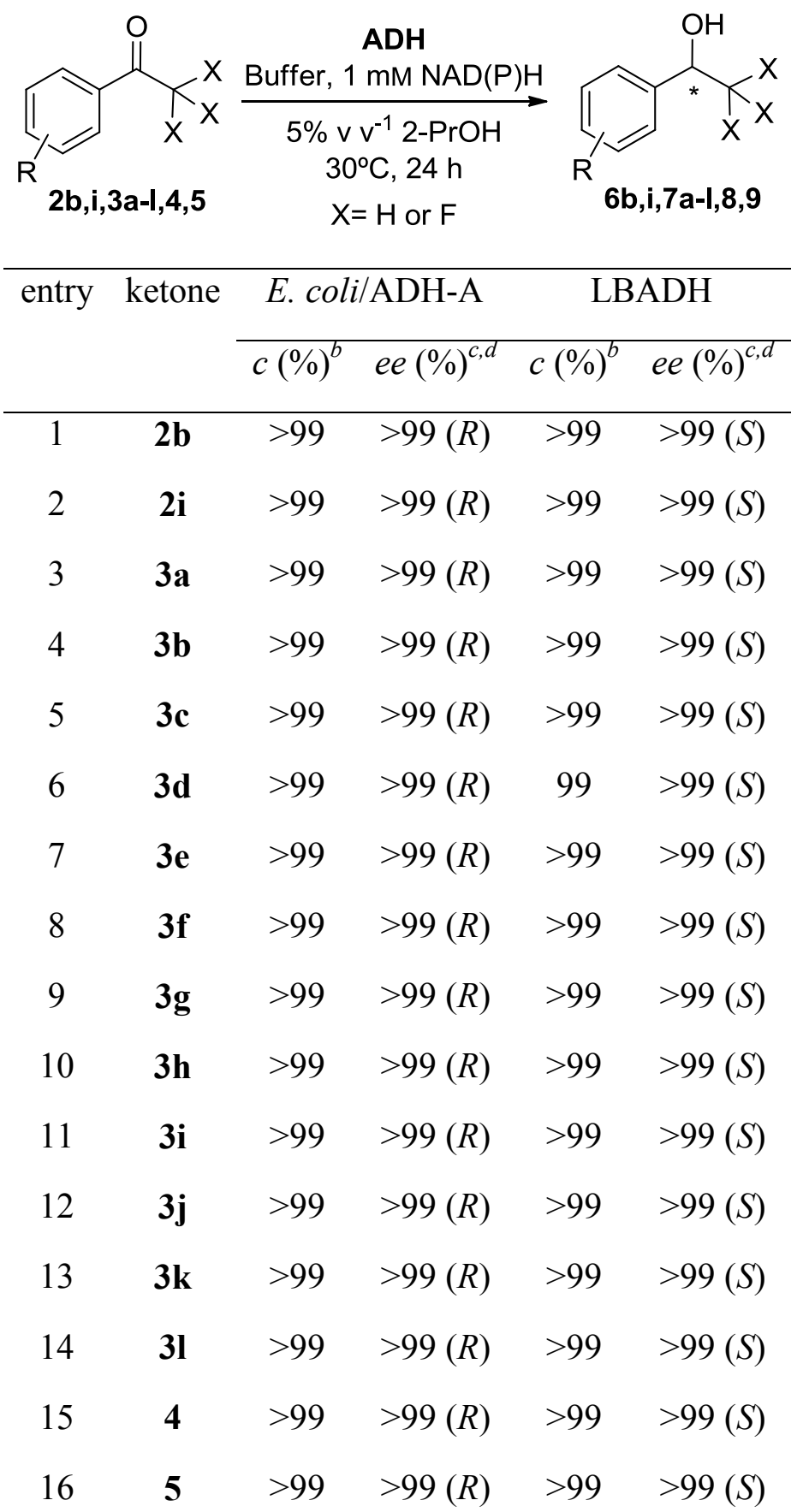

${ }^{a}$ For experimental details, see Experimental Section. ${ }^{b}$ Measured by GC. ${ }^{c}$ Measured by chiral GC. ${ }^{d}$ Change in Cahn-Ingold-Prelog (CIP) priority. 
Gratefully, in every case the substrate was completely transformed into the enantiopure $(R)$ - or $(S)$ alcohol when using ADH-A or $\mathrm{LBADH}$, independently it bore electron withdrawing or electron donating groups at meta- or para-positions. Also di- or trifluorinated ketones $\mathbf{4}$ and $\mathbf{5}$ afforded perfect conversions and ee with both biocatalysts.

One additional advantage of working with overexpressed ADH preparations is the possibility to avoid the use of external cofactor when performing the bioreductions. ${ }^{19}$ Therefore, some of these ketones were tested at higher substrate concentration with E. coli/ADH-A without the addition of external NADH (Table 2). It was interesting to observe that in some cases the enantiopurity of the final alcohols slightly decreased. This could be probably due to the action of other NADP-dependent ADHs from the host organism that in the absence of additional NADH could compete with ADH-A diminishing the overall selectivity. Moreover, it was demonstrated that even at high concentrations such as $200 \mathrm{mM}$, conversions remained quantitative.

Table 2. Bioreductions of fluorinated ketones using E. coli/ADH-A and 2-PrOH $\left(10 \% \mathrm{v} \mathrm{v}^{-1}\right)$ without the addition of external NADH $(t=24 \mathrm{~h})^{a}$

\begin{tabular}{ccccc}
\hline entry & ketone & concentration $(\mathrm{mM})$ & $c(\%)^{b}$ & $e e(\%)^{c, d}$ \\
\hline 1 & 3a & 200 & $>99$ & $>99(R)$ \\
2 & 3b & 200 & $>99$ & $97(R)$ \\
3 & 3c & 200 & $>99$ & $98(R)$ \\
4 & $\mathbf{3 e}$ & 200 & $>99$ & $>99(R)$ \\
5 & $\mathbf{4}$ & 200 & $>99$ & $94(R)$ \\
6 & $\mathbf{5}$ & 100 & $>99$ & $>99(R)$
\end{tabular}

${ }^{a}$ For experimental details, see Experimental Section. ${ }^{b}$ Measured by GC. ${ }^{c}$ Measured by chiral GC. ${ }^{d}$ Change in CIP priority.

In a next step, we decided to increase the substrate concentration keeping constant the quantity of LBADH in the presence of $1 \mathrm{mM}$ of the nicotinamide cofactor and just increasing the amount of 2- 
$\mathrm{PrOH}$, although due to the fact that $\alpha$-halogenated ketones can be quasi-irreversibly reduced ${ }^{20}$ it was not necessary a huge excess of the hydrogen donor. A selection of the results obtained is shown in Figure 1.<smiles>O[C@H](CBr)c1ccc(F)cc1</smiles>

6b, $c=74 \%$ ee $>99 \%(S)$<smiles>O[C@H](CF)c1ccc(Cl)cc1</smiles>

7c, $c>99 \%$ ee $>99 \%(S)$<smiles>COc1ccc([C@@H](O)CF)cc1</smiles>

$7 \mathrm{~g}, c=88 \%$ ee $>99 \%(S)$<smiles>O[C@H](CF)c1cccc(Cl)c1</smiles>

$7 \mathbf{j}, c>99 \%$ ee $>99 \%(S)$<smiles>O[C@H](CF)c1ccccc1</smiles>

$7 a, c>99 \%$ ee $>99 \%$ (S)<smiles>O[C@H](CF)c1ccc(Br)cc1</smiles>

7d, $c>99 \%$ ee $>99 \%(S)$<smiles>O=[N+]([O-])c1ccc([C@@H](O)CF)cc1</smiles>

$7 \mathrm{~h}, c=96 \%$ ee $>99 \%$ (S)<smiles>O[C@H](c1ccccc1)C(F)F</smiles>

8, c >99\% ee $>99 \%(S)$<smiles>O[C@H](CF)c1ccc(F)cc1</smiles>

7b, c >99\% ee $>99 \%$ (S)<smiles>Cc1ccc([C@@H](O)CF)cc1</smiles>

7f, $c>99 \%$ ee $>99 \%$ (S)<smiles>O[C@H](CF)c1cccc(F)c1</smiles>

7i, c >99\% ee $>99 \%$ (S)<smiles>O[C@H](c1ccccc1)C(F)(F)F</smiles>

9, c >99\% ee $>99 \%(S)$

Figure 1. Examples of enantiopure fluorinated alcohols obtained at $0.5 \mathrm{M}$ substrate concentration with LBADH and 2-PrOH ( $t=24 \mathrm{~h})$. For other results, see Supporting Information.

Several enantiopure fluorinated alcohols were achieved at $0.5 \mathrm{M}$ concentration with excellent conversions in the presence of $3 \mathrm{U}$ of the enzyme and $1 \mathrm{mM} \mathrm{NADP}^{+}$. In these examples, $20 \% \mathrm{v} \mathrm{v}^{-1}$ of the hydrogen donor 2-PrOH was employed, approximately a molar excess of 5:1 with regards to the ketone substrate, confirming that the bioreduction of these derivatives was thermodynamically favored.

$(R)-6 \mathbf{b}$ is a precursor of cholesterol absorption inhibitors (CAI) such as AZD4121 or ezetimibe (Scheme 2), a class of compounds that have attracted attention for the treatment of hypercholesterolemia, related to several diseases as atherosclerosis. ${ }^{21}$ This alcohol was recently 
synthesized via asymmetric reduction with $(R)$-2-methyloxazaborolidine in the presence of boranedimethyl sulfide complex in THF at $0^{\circ} \mathrm{C} .{ }^{22}$ With regards to biocatalyzed synthesis, Zhu and co-workers obtained the enantiopure $(S)$-alcohol using an isolated ADH from Candida magnolia with glucose and GDH to recycle the nicotinamide cofactor. ${ }^{23}$

Scheme 2. $(R)-6 \mathbf{b}$, a precursor of cholesterol absorption inhibitors such as ezetimibe

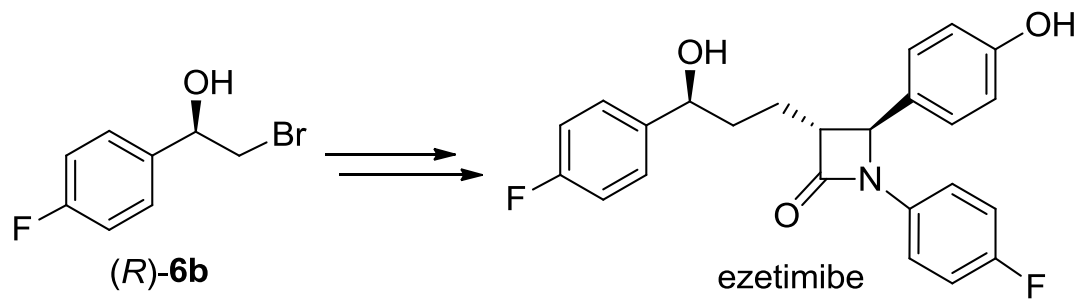

Due to the relevance of this bromohydrin, the $E$. coli/ADH-A catalyzed bioreduction of $\mathbf{2 b}$ was performed at $260 \mathrm{mg}$-scale with a $100 \mathrm{mM}$ substrate concentration, obtaining enantiopure $(R)-6 \mathbf{b}$ after purification with $70 \%$ yield.

Overall, with the systematic study shown here it was demonstrated that ADH-catalyzed hydrogen transfer reduction of $\alpha$-fluoro ketones is a very convenient method to synthesize both alcohol antipodes in an enantiopure form at high substrate concentrations with excellent conversions. Thus, when performing the bioreduction in the presence of overexpressed enzymes it was also possible to avoid the use of external nicotinamide cofactor. Due to the nature of these activated ketones, it was not necessary the employment of a huge excess of the hydrogen donor.

\section{Experimental Section}

General. Acetophenones 1e, 1i, 1j, 1k, and 1l, $\alpha$-bromoacetophenones $\mathbf{2 b}, \mathbf{2 c}, \mathbf{2 d}, \mathbf{2 f}, \mathbf{2 g}$, and $\mathbf{2 h}$, difluorinated ketone $\mathbf{4}$ and trifluorinated derivative $\mathbf{5}$ were obtained from commercial sources. All other reagents and solvents were of the highest quality available. LBADH from Lactobacillus brevis and GDH were obtained from commercial sources. The following overexpressed enzymes in E. coli have been 
kindly provided by Prof. Wolfgang Kroutil from the University of Graz (Austria): ADH-A from Rhodococcus ruber (E. coli/ADH-A), ADH-T from Thermoanaerobium sp. (E. coli/ADH-T), SyADH from Sphingobium yanoikuyae (E. coli/SyADH), RasADH from Ralstonia sp. (E. coli/RasADH), and TeSADH from Thermoanaerobacter ethanolicus (E. coli/TeSADH).

Chemical reactions were monitored by analytical TLC, performed on silica gel 60 F254 plates and visualized by UV irradiation. Flash chromatography was performed using silica gel 60 (230-400 mesh). Melting points were taken on samples in open capillary tubes and are uncorrected. IR spectra were recorded on an infrared Fourier transform spectrophotometer on $\mathrm{NaCl}$ pellets. ${ }^{1} \mathrm{H}-,{ }^{13} \mathrm{C},{ }^{19} \mathrm{~F}-\mathrm{NMR}$, and DEPT were obtained using 300 and $400 \mathrm{MHz}$ spectrometers for routine experiments. The chemical shifts $(\delta)$ are given in ppm and the coupling constants $(J)$ in Hertz $(\mathrm{Hz})$. $\mathrm{ESI}^{+}$mode was used to record mass spectra (MS) and ESI-TOF for HRMS. Gas chromatography (GC) analyses were performed on a standard GC chromatograph. Calibration curves were performed with $\alpha$-fluoro ketones and the corresponding fluorohydrins to measure accurately the enzymatic conversions. Optical rotations were measured using a standard polarimeter and are quoted in units of $10^{-1} \mathrm{deg} \mathrm{cm}^{2} \mathrm{~g}^{-1}$. For new compounds, the indirect assignation of their configuration was based in two convergent criteria: a) the perfect stereoselectivity shown by ADH-A and LBADH for the other members of this fluorinated family and other similar compounds, and b) the order of the elution peaks in the chiral GC column.

Synthesis of $\alpha$-bromoacetophenone derivatives 2. To a solution of the acetophenone derivative (15.0 mmol, 1 equiv) in $8 \mathrm{~mL}$ of acetonitrile, NBS (2.72 $\mathrm{g}, 15.3 \mathrm{mmol}, 1.02$ equiv) and $p$ toluenesulfonic acid (2.85 g, $15.0 \mathrm{mmol}, 1$ equiv) were added. The reaction mixture was stirred for $24 \mathrm{~h}$ at $50^{\circ} \mathrm{C}$. After that time, the solvent was evaporated under reduced pressure. Then, a water solution of saturated $\mathrm{NaHCO}_{3}(30 \mathrm{~mL})$ was added and the solution was extracted with dichloromethane $(3 \times 30$ $\mathrm{mL}$ ). The organic layers were combined and dried over $\mathrm{Na}_{2} \mathrm{SO}_{4}$. The solvent was evaporated and the residue was subjected to column chromatography (silica gel) using hexanes $/ \mathrm{CH}_{2} \mathrm{Cl}_{2}$ (from 9:1 to 4:1) as eluent. These compounds exhibited physical and spectral data in agreement with those reported. ${ }^{24} \mathbf{2 e}$ 
(4.30 g, 88\% yield), $2 \mathbf{i}(2.12 \mathrm{~g}, 66 \%$ yield), $\mathbf{2 j}$ (2.88 g, 82\% yield), $2 \mathbf{k}$ (2.45 g, 71\% yield), and $2 \mathbf{l}(2.60$ g, $71 \%$ yield).

Synthesis of $\boldsymbol{\alpha}$-fluoroacetophenone derivatives 3 . A mixture of $\mathrm{KF}$ (4.8 mmol, 1.5 equiv), $\mathrm{ZnF}_{2}$ (4.8 mmol, 1.5 equiv) in $7 \mathrm{~mL}$ of acetonitrile was stirred and heated at $100^{\circ} \mathrm{C}$ in a sealed heavy-walled Pyrex tube for $1 \mathrm{~h}$. Then, a solution of the substrate $(3.2 \mathrm{mmol}, 1.0$ equiv) in acetonitrile $(7 \mathrm{~mL})$ was added into the reaction mixture. Then it was heated for $24 \mathrm{~h}$ at $100^{\circ} \mathrm{C}$. After that time, the solvent was evaporated under reduced pressure. Then, water $(20 \mathrm{~mL})$ was added and the solution was extracted with dichloromethane $(3 \times 20 \mathrm{~mL})$. The organic layers were combined and dried over $\mathrm{Na}_{2} \mathrm{SO}_{4}$. The solvent was evaporated and the residue was subjected to column chromatography (silica gel) using hexanes/EtOAc (from 95:5 to 90:10) as eluent. These compounds exhibited physical and spectral data in agreement with those reported. ${ }^{14,25} \mathbf{3 b}(230 \mathrm{mg}, 47 \%$ yield), 3c (255 mg, 47\% yield), 3d (625 mg, $90 \%$ yield), 3e (326 mg, 39\% yield), 3f (274 mg, 56\% yield), 3g (380 mg, 71\% yield), $3 \mathbf{h}$ (210 mg, 36\% yield), 3i (265 mg, 53\% yield), 3j (367 mg, 66\% yield), 3k (368 mg, 68\% yield), and $3 \mathbf{l}$ (214 mg, 36\% yield).

2-Fluoro-4'-iodoacetophenone (3e). White solid; mp: 92.4-95.3 C; IR ( $\mathrm{NaCl})$ : 3054, 2987, 1422 , 1265, 972, 896, 741, $666 \mathrm{~cm}^{-1} ;{ }^{1} \mathrm{H}$ NMR $\left(300 \mathrm{MHz}, \mathrm{CDCl}_{3}\right): \delta 7.89(\mathrm{~d}, J=8.6 \mathrm{~Hz}, 2 \mathrm{H}), 7.63(\mathrm{~d}, J=8.4$ $\mathrm{Hz}, 2 \mathrm{H}), 5.49(\mathrm{~d}, J=46.9 \mathrm{~Hz}, 2 \mathrm{H}) ;{ }^{13} \mathrm{C} \mathrm{NMR}\left(75 \mathrm{MHz}, \mathrm{CDCl}_{3}\right): \delta 193.0(\mathrm{~d}, J=15.8 \mathrm{~Hz}), 138.3,133.0$,

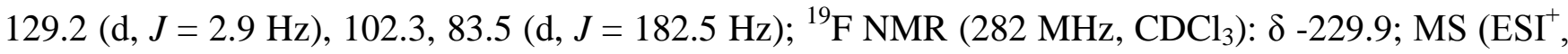
$m / z): 287\left[(\mathrm{M}+\mathrm{Na})^{+}, 100 \%\right], 265\left[(\mathrm{M}+\mathrm{H})^{+}, 30 \%\right] ;$ HRMS $\left(\mathrm{ESI}^{+}, m / z\right)$ calculated for $\mathrm{C}_{8} \mathrm{H}_{6} \mathrm{OFINa}$ $(\mathrm{M}+\mathrm{Na})^{+}: 286.9365$, found: 286.9340 .

3'-Chloro-2-fluoroacetophenone (3j). Yellow oil; IR (NaCl): 3057, 2987, 2933, 1711, 1575, 1428, 1266, 1229, 1101, 1087, 998, 739, $704 \mathrm{~cm}^{-1} ;{ }^{1} \mathrm{H}$ NMR (300 MHz, $\left.\mathrm{CDCl}_{3}\right): \delta 7.91(\mathrm{~m}, 1 \mathrm{H}), 7.79(\mathrm{~m}, 1 \mathrm{H})$, $7.62(\mathrm{~m}, 1 \mathrm{H}), 7.47(\mathrm{~m}, 1 \mathrm{H}), 5.51(\mathrm{~d}, J=46.8 \mathrm{~Hz}, 2 \mathrm{H}) ;{ }^{13} \mathrm{C} \mathrm{NMR}\left(75 \mathrm{MHz}, \mathrm{CDCl}_{3}\right): \delta 192.4(\mathrm{~d}, J=16.0$ $\mathrm{Hz}), 135.2(\mathrm{~d}, J=11.6 \mathrm{~Hz}), 134.1,130.3,128.1(\mathrm{~d}, J=2.9 \mathrm{~Hz}), 126.0(\mathrm{~d}, J=2.7 \mathrm{~Hz}), 83.6(\mathrm{~d}, J=182.7$

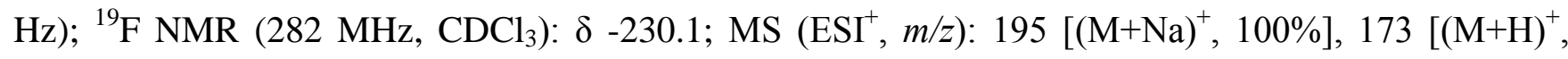
70\%]; HRMS (ESI $\left.{ }^{+}, m / z\right)$ calculated for $\mathrm{C}_{8} \mathrm{H}_{6} \mathrm{OClFNa}(\mathrm{M}+\mathrm{Na})^{+}:$194.9992, found: 194.9983 . 
2-Fluoro-3'-methoxyacetophenone (3k). White solid; mp: 55.7-58.8 ${ }^{\circ}$; IR ( $\left.\mathrm{NaCl}\right)$ : 3054, 2987, $1710,1600,1584,1432,1265,1092,738,705 \mathrm{~cm}^{-1} ;{ }^{1} \mathrm{H}$ NMR $\left(300 \mathrm{MHz}, \mathrm{CDCl}_{3}\right): \delta 7.42(\mathrm{~m}, 3 \mathrm{H}), 7.18$ $(\mathrm{m}, 1 \mathrm{H}), 5.54(\mathrm{~d}, J=46.9 \mathrm{~Hz}, 2 \mathrm{H}), 3.88(\mathrm{~s}, 3 \mathrm{H}) ;{ }^{13} \mathrm{C} \mathrm{NMR}\left(75 \mathrm{MHz}, \mathrm{CDCl}_{3}\right): \delta 193.2(\mathrm{~d}, J=15.2 \mathrm{~Hz})$, $160.0,134.9,129.9,120.7,120.2(\mathrm{~d}, J=2.3 \mathrm{~Hz}), 112.1(\mathrm{~d}, J=2.2 \mathrm{~Hz}), 83.5(\mathrm{~d}, J=181.4 \mathrm{~Hz}), 55.5 ;{ }^{19} \mathrm{~F}$

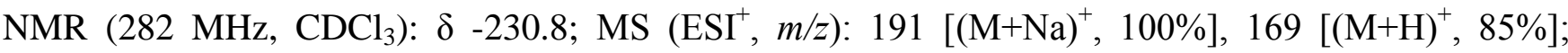
HRMS $\left(\mathrm{ESI}^{+}, m / z\right)$ calculated for $\mathrm{C}_{9} \mathrm{H}_{9} \mathrm{O}_{2} \mathrm{FNa}(\mathrm{M}+\mathrm{Na})^{+}: 191.0488$, found: 191.0479 .

General procedure for the synthesis of the racemic alcohols 6-9. To a solution of the corresponding ketone $(4.0 \mathrm{mmol})$ in methanol $(5 \mathrm{~mL})$ at $0^{\circ} \mathrm{C}$, sodium borohydride $(1.2 \mathrm{mmol})$ was added. When the reduction was completed (according to the TLC), a few drops of $1 \mathrm{M} \mathrm{HCl}$ were added. The solvent was evaporated under reduced pressure. Then, water $(10 \mathrm{~mL})$ was added and the solution was extracted with dichloromethane $(3 \times 10 \mathrm{~mL})$. The organic layers were combined and dried over $\mathrm{Na}_{2} \mathrm{SO}_{4}$. The solvent was evaporated and the residue was subjected to column chromatography (silica gel) using mixtures of hexanes/ethyl acetate (from 9:1 to 4:1) as eluents. These compounds exhibited physical and spectral data in agreement with those reported. ${ }^{23,26}$ Isolated yields: $71-95 \%$.

2-Bromo-1-(3-fluorophenyl)ethanol (6i). White solid; mp: 66.8-69.0 C; IR ( $\mathrm{NaCl})$ : 3054, 2987, 1422, 1265, 1158, 896, 740, $705 \mathrm{~cm}^{-1} ;{ }^{1} \mathrm{H}$ NMR $\left(300 \mathrm{MHz}, \mathrm{CDCl}_{3}\right): \delta 7.37(\mathrm{~m}, 1 \mathrm{H}), 7.16(\mathrm{~m}, 2 \mathrm{H}), 7.05$ $(\mathrm{tdd}, J=8.4,2.5,0.9 \mathrm{~Hz}, 1 \mathrm{H}), 4.95(\mathrm{dd}, J=8.8,3.3 \mathrm{~Hz}, 1 \mathrm{H}), 3.65(\mathrm{dd}, J=10.5,3.3 \mathrm{~Hz}, 1 \mathrm{H}), 3.55(\mathrm{dd}, J$ $=10.5,8.8 \mathrm{~Hz}, 1 \mathrm{H}), 2.69(\mathrm{br} \mathrm{s}, 1 \mathrm{H}) ;{ }^{13} \mathrm{C} \mathrm{NMR}\left(75 \mathrm{MHz}, \mathrm{CDCl}_{3}\right): \delta 163.0(\mathrm{~d}, J=245.3 \mathrm{~Hz}), 142.8(\mathrm{~d}, J$ $=27.4 \mathrm{~Hz}), 130.2(\mathrm{~d}, J=31.8 \mathrm{~Hz}), 121.6(\mathrm{~d}, J=11.4 \mathrm{~Hz}), 115.3(\mathrm{~d}, J=83.7 \mathrm{~Hz}), 113.0(\mathrm{~d}, J=89.0 \mathrm{~Hz})$, 73.1, 39.9; ${ }^{19} \mathrm{~F} \mathrm{NMR}\left(282 \mathrm{MHz}, \mathrm{CDCl}_{3}\right): \delta-112.2 ;[\alpha]_{\mathrm{D}}{ }^{20}=+41.0\left(\mathrm{c} 1.13, \mathrm{CHCl}_{3}\right), e e>99 \%(S) .{ }^{27}$

1-(4-Chlorophenyl)-2-fluoroethanol $(7 \mathrm{c}) .^{26 \mathrm{~d}}[\alpha]_{\mathrm{D}}^{20}=+42.9\left(\mathrm{c} 1.67, \mathrm{CHCl}_{3}\right)$, ee >99\% $(S)$.

2-Fluoro-1-(4-iodophenyl)ethanol (7e). Light yellow solid; mp: 42.3-45.0 C; IR (NaCl): 3055, 2987, 1422, 1265, 896, 740, $705 \mathrm{~cm}^{-1} ;{ }^{1} \mathrm{H}$ NMR $\left(300 \mathrm{MHz}, \mathrm{CDCl}_{3}\right): \delta 7.73(\mathrm{~d}, J=8.4 \mathrm{~Hz}, 2 \mathrm{H}), 7.17(\mathrm{~d}, J=$ $8.2 \mathrm{~Hz}, 2 \mathrm{H}), 5.00(\mathrm{~m}, 1 \mathrm{H}), 4.60-4.28(\mathrm{~m}, 2 \mathrm{H}), 2.52$ (br s, $1 \mathrm{H}) ;{ }^{13} \mathrm{C} \mathrm{NMR}\left(75 \mathrm{MHz}, \mathrm{CDCl}_{3}\right): \delta 137.8$, 137.7, 128.2, 94.0, $86.8(\mathrm{~d}, J=173.9 \mathrm{~Hz}), 72.4(\mathrm{~d}, J=20.1 \mathrm{~Hz}) ;{ }^{19} \mathrm{~F}$ NMR $\left(282 \mathrm{MHz}, \mathrm{CDCl}_{3}\right): \delta-229.9$; 
MS $\left(\mathrm{ESI}^{+}, \mathrm{m} / z\right): 289\left[(\mathrm{M}+\mathrm{Na})^{+}, 100 \%\right], 249\left[(\mathrm{M}-\mathrm{OH})^{+}, 25 \%\right] ; \mathrm{HRMS}\left(\mathrm{ESI}^{+}, \mathrm{m} / z\right)$ calculated for $\mathrm{C}_{8} \mathrm{H}_{8} \mathrm{OFINa}(\mathrm{M}+\mathrm{Na})^{+}: 288.9484$, found: $288.9496 ;[\alpha]_{\mathrm{D}}^{20}=+30.3\left(\mathrm{c} 1.04, \mathrm{CHCl}_{3}\right), e e>99 \%(S)$.

2-Fluoro-1-(4-methylphenyl)ethanol (7f). White solid; mp: 33.8-37.5º ; IR (NaCl): 3054, 2987, 1422, 1265, 1090, 1004, 896, 743, $705 \mathrm{~cm}^{-1} ;{ }^{1} \mathrm{H}$ NMR $\left(300 \mathrm{MHz}, \mathrm{CDCl}_{3}\right): \delta 7.31(\mathrm{~d}, J=8.1 \mathrm{~Hz}, 2 \mathrm{H})$, $7.20(\mathrm{~d}, J=8.0 \mathrm{~Hz}, 2 \mathrm{H}), 5.02(\mathrm{~m}, 1 \mathrm{H}), 4.61-4.41(\mathrm{~m}, 2 \mathrm{H}), 2.50(\mathrm{dd}, J=2.9,0.8 \mathrm{~Hz}, 1 \mathrm{H}), 2.38(\mathrm{~s}, 3 \mathrm{H})$; ${ }^{13} \mathrm{C}$ NMR $\left(75 \mathrm{MHz}, \mathrm{CDCl}_{3}\right): \delta 138.3,135.1(\mathrm{~d}, J=8.2 \mathrm{~Hz}), 129.3,126.3,87.2(\mathrm{~d}, J=173.2 \mathrm{~Hz}), 72.8$ $(\mathrm{d}, J=19.6 \mathrm{~Hz}), 21.2 ;{ }^{19} \mathrm{~F}$ NMR $\left(282 \mathrm{MHz}, \mathrm{CDCl}_{3}\right): \delta-220.4 ; \mathrm{MS}\left(\mathrm{ESI}^{+}, \mathrm{m} / z\right): 177\left[(\mathrm{M}+\mathrm{Na})^{+}, 100 \%\right]$ $137\left[(\mathrm{M}-\mathrm{OH})^{+}, 90 \%\right]$; HRMS $\left(\mathrm{ESI}^{+}\right)$: calculated for $\mathrm{C}_{9} \mathrm{H}_{11} \mathrm{OFNa}(\mathrm{M}+\mathrm{Na})^{+}:$177.0675, found: 177.0686; $[\alpha]_{\mathrm{D}}^{20}=+51.9\left(\mathrm{c} 1.34, \mathrm{CHCl}_{3}\right), e e>99 \%(S)$.

2-Fluoro-1-(3-fluorophenyl)ethanol (7i). Light yellow oil; IR (NaCl): 3055, 2986, 1594, 1450, 1266, 1137, 1010, 896, 739, $705 \mathrm{~cm}^{-1} ;{ }^{1} \mathrm{H}$ NMR $\left(300 \mathrm{MHz}, \mathrm{CDCl}_{3}\right): \delta 7.37$ (m, 1H), $7.16(\mathrm{~m}, 2 \mathrm{H}), 7.05$ (tdd, $J$ $=8.4,2.5,1.0 \mathrm{~Hz}, 1 \mathrm{H}), 5.05(\mathrm{~m}, 1 \mathrm{H}), 4.64-4.31(\mathrm{~m}, 2 \mathrm{H}), 2.57($ br s, $1 \mathrm{H}) ;{ }^{13} \mathrm{C} \mathrm{NMR}\left(75 \mathrm{MHz}, \mathrm{CDCl}_{3}\right): \delta$ $163.0(\mathrm{~d}, J=245.1 \mathrm{~Hz}), 140.7(\mathrm{~d}, J=7.5 \mathrm{~Hz}), 130.2(\mathrm{~d}, J=8.1 \mathrm{~Hz}), 121.9(\mathrm{~d}, J=2.7 \mathrm{~Hz}), 115.3(\mathrm{~d}, J=$ $21.0 \mathrm{~Hz}), 113.4(\mathrm{~d}, J=22.2 \mathrm{~Hz}), 86.9(\mathrm{~d}, J=173.6 \mathrm{~Hz}), 72.3(\mathrm{~d}, J=20.1 \mathrm{~Hz}) ;{ }^{19} \mathrm{~F} \mathrm{NMR}(282 \mathrm{MHz}$, $\left.\mathrm{CDCl}_{3}\right): \delta-112.4,-221.5 ;[\alpha]_{\mathrm{D}}{ }^{20}=+42.3\left(\mathrm{c} 1.21, \mathrm{CHCl}_{3}\right), e e>99 \%(S){ }^{27}$

1-(3-Chlorophenyl)-2-fluoroethanol (7j). Yellow oil; IR (NaCl): 3060, 2983, 2952, 1600, 1576, 1479, 1433, 1266, 1198, 1079, 1012, 912, 789, 739, $703 \mathrm{~cm}^{-1} ;{ }^{1} \mathrm{H}$ NMR (300 MHz, $\left.\mathrm{CDCl}_{3}\right): \delta 7.43(\mathrm{~m}$, 1H), $7.30(\mathrm{~m}, 3 \mathrm{H}), 5.06(\mathrm{~m}, 1 \mathrm{H}), 4.63-4.30(\mathrm{~m}, 2 \mathrm{H}), 2.64(\mathrm{br} \mathrm{s}, 1 \mathrm{H}) ;{ }^{13} \mathrm{C} \mathrm{NMR}\left(75 \mathrm{MHz}, \mathrm{CDCl}_{3}\right): \delta$ $140.1(\mathrm{~d}, J=8.0 \mathrm{~Hz}), 134.6,129.9,128.6,126.5,124.5,86.9(\mathrm{~d}, J=173.7 \mathrm{~Hz}), 72.3(\mathrm{~d}, J=20.0 \mathrm{~Hz})$; ${ }^{19} \mathrm{~F}$ NMR $\left(282 \mathrm{MHz}, \mathrm{CDCl}_{3}\right): \delta-221.4 ;[\alpha]_{\mathrm{D}}{ }^{20}=+42.1\left(\mathrm{c} 1.65, \mathrm{CHCl}_{3}\right), e e>99 \%(S) .{ }^{27}$

2-Fluoro-1-(3-methoxyphenyl)ethanol (7k). Light yellow oil; IR (NaCl): 3056, 2950, 2838, 1603, $1587,1489,1467,1456,1436,1320,1267,1158,1043,1010,881,786,737,700 \mathrm{~cm}^{-1} ;{ }^{1} \mathrm{H}$ NMR (300 $\left.\mathrm{MHz}, \mathrm{CDCl}_{3}\right): \delta 7.31(\mathrm{~m}, 1 \mathrm{H}), 6.97(\mathrm{~m}, 2 \mathrm{H}), 6.89(\mathrm{~m}, 1 \mathrm{H}), 5.02(\mathrm{~m}, 1 \mathrm{H}), 4.63-4.32(\mathrm{~m}, 2 \mathrm{H}), 3.84(\mathrm{~s}$, 3H), 2.39 (br s, $1 \mathrm{H}) ;{ }^{13} \mathrm{C} \mathrm{NMR}\left(75 \mathrm{MHz} \mathrm{CDCl}_{3}\right): \delta 159.9,139.7(\mathrm{~d}, J=8.2 \mathrm{~Hz}), 129.7,118.5,113.9$, 111.8, 87.2 (d, $J=173.3 \mathrm{~Hz}), 72.9(\mathrm{~d}, J=19.7 \mathrm{~Hz}), 55.3 ;{ }^{19} \mathrm{~F}$ NMR $\left(282 \mathrm{MHz}, \mathrm{CDCl}_{3}\right): \delta-220.7 ; \mathrm{MS}$ 
$\left(\mathrm{ESI}^{+}, \mathrm{m} / \mathrm{z}\right): 193\left[(\mathrm{M}+\mathrm{Na})^{+}, 100 \%\right], 153\left[(\mathrm{M}-\mathrm{OH})^{+}, 50 \%\right] ; \mathrm{HRMS}\left(\mathrm{ESI}^{+}, \mathrm{m} / z\right)$ calculated for $\mathrm{C}_{9} \mathrm{H}_{11} \mathrm{O}_{2} \mathrm{FNa}(\mathrm{M}+\mathrm{Na})^{+}:$193.0637, found: $193.0635 ;[\alpha]_{\mathrm{D}}{ }^{20}=+42.3\left(\mathrm{c} 1.85, \mathrm{CHCl}_{3}\right), e e>99 \%(S)$.

2-Fluoro-1-(3-nitrophenyl)ethanol (7l). Light yellow solid; mp: 49.9-54.8 C; IR (NaCl): 3054, 2987, 1534, 1422, 1353, 1265, 896, 738, $705 \mathrm{~cm}^{-1} ;{ }^{1} \mathrm{H} \mathrm{NMR}\left(300 \mathrm{MHz}, \mathrm{CDCl}_{3}\right): \delta 8.32($ ap t, $J=2.0 \mathrm{~Hz}, 1 \mathrm{H})$, $8.22(\mathrm{ddd}, J=8.2,2.3,1.0 \mathrm{~Hz}, 1 \mathrm{H}), 7.77(\mathrm{~d}, J=7.7 \mathrm{~Hz}, 1 \mathrm{H}), 7.59($ ap t, $J=8.0 \mathrm{~Hz}, 1 \mathrm{H}), 5.16(\mathrm{~m}, 1 \mathrm{H})$, 4.69-4.36 (m, 2H), 2.76 (br s, $1 \mathrm{H}) ;{ }^{13} \mathrm{C} \mathrm{NMR} \mathrm{(75} \mathrm{MHz,} \mathrm{CDCl}_{3}$ ): $\delta 148.4,140.4(\mathrm{~d}, J=7.6 \mathrm{~Hz}), 132.4$, 129.6, 123.3, 121.4, $86.5(\mathrm{~d}, J=174.2 \mathrm{~Hz}), 71.9(\mathrm{~d}, J=20.5 \mathrm{~Hz}) ;{ }^{19} \mathrm{~F} \mathrm{NMR}\left(282 \mathrm{MHz}, \mathrm{CDCl}_{3}\right): \delta$

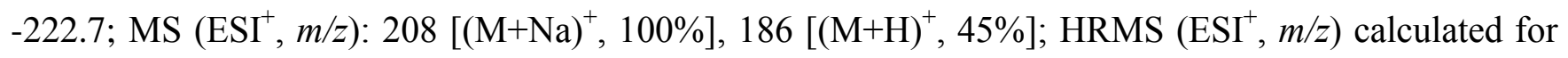
$\mathrm{C}_{8} \mathrm{H}_{8} \mathrm{NO}_{3} \mathrm{FNa}(\mathrm{M}+\mathrm{Na})^{+}: 208.0374$, found: 208.0380; $[\alpha]_{\mathrm{D}}{ }^{20}=+33.5\left(\mathrm{c} 1.04, \mathrm{CHCl}_{3}\right), e e>99 \%(S)$.

Enzymatic screening with 3a. In a $1.5 \mathrm{~mL}$ Eppendorf vial, LBADH (3 U) or lyophilized cells of the overexpressed ADH (ADH-A, ADH-T, RasADH, SyADH, or TeSADH) in E. coli (20 mg), were added in Tris. $\mathrm{HCl}$ buffer $50 \mathrm{mM}$ pH $7.5\left(570 \mu \mathrm{L}, 1 \mathrm{mM} \mathrm{NADP}^{+}\right.$for LBADH, ADH-T, RasADH, SyADH, and TeSADH or $1 \mathrm{mM} \mathrm{NADH}$ for ADH-A; $1 \mathrm{mM} \mathrm{MgCl}_{2}$ for $\left.\mathrm{LBADH}\right)$, and mixed with $3 \mathbf{a}(30 \mathrm{mM})$ and 2propanol (30 $\mu \mathrm{L}, 5 \% \mathrm{v} \mathrm{v}^{-1}$, for LBADH, ADH-A, ADH-T, SyADH, and TeSADH) or with glucose (1.5 $\mathrm{M})$ and $\mathrm{GDH}(5 \mathrm{U})$ for RasADH. The reactions were shaken at $30^{\circ} \mathrm{C}$ and $250 \mathrm{rpm}$ for $24 \mathrm{~h}$ and stopped by extraction with ethyl acetate $(2 \times 0.5 \mathrm{~mL})$. The organic layers were separated by centrifugation $(2$ min, $13000 \mathrm{rpm}$ ) and dried over $\mathrm{Na}_{2} \mathrm{SO}_{4}$. Conversions of the corresponding alcohol were determined by GC obtaining the following results: for $\mathrm{LBADH}(>99 \%)$, E. coli/ADH-A (>99\%), E. coli/RasADH (99\%), E. coli/SyADH (4\%), E. coli/ADH-T (99\%), and E. coli/TeSADH (64\%).

General procedure for an enzymatic reduction using LBADH from Lactobacillus brevis. In a 1.5 mL Eppendorf vial, LBADH (3 U) was added in Tris.HCl buffer $50 \mathrm{mM} \mathrm{pH} 7.5$ (570 $\mu \mathrm{L}, 1 \mathrm{mM} \mathrm{NADP}^{+}$, $\left.1 \mathrm{mM} \mathrm{MgCl}_{2}\right)$ and mixed with 2-propanol $\left(30 \mu \mathrm{L}, 5 \% \mathrm{v} \mathrm{v}^{-1}\right)$ and the corresponding ketone (30 or 50 $\mathrm{mM}$ ). The reaction was shaken at $30^{\circ} \mathrm{C}$ and $250 \mathrm{rpm}$ for $24 \mathrm{~h}$ and stopped by extraction with ethyl acetate $(2 \times 0.5 \mathrm{~mL})$. The organic layer was separated by centrifugation ( $2 \mathrm{~min}, 13000 \mathrm{rpm})$ and dried over $\mathrm{Na}_{2} \mathrm{SO}_{4}$. Conversions and $e e$ of the corresponding alcohols were determined by GC (see Tables 1 
and S1). For $\alpha$-brominated ketones, in order to avoid undesired $\mathrm{S}_{\mathrm{N}} 2$ reactions with Tris.HCl buffer, ${ }^{13}$ Tris. $\mathrm{H}_{2} \mathrm{SO}_{4}$ buffer $50 \mathrm{mM}$ pH $7.5\left(570 \mu \mathrm{L}, 1 \mathrm{mM} \mathrm{NADP}{ }^{+}, 1 \mathrm{mM} \mathrm{MgBr} 2\right)$ was used.

General procedure for an enzymatic reduction using $E$. coli/ADH-A from Rhodococcus ruber. In a $1.5 \mathrm{~mL}$ Eppendorf vial, E. coli/ADH-A $(20 \mathrm{mg})$ was added in Tris-HCl buffer $50 \mathrm{mM} \mathrm{pH} 7.5(570 \mu \mathrm{L}$, $1 \mathrm{mM}$ NADH) and mixed with 2-propanol $\left(30 \mu \mathrm{L}, 5 \% \mathrm{v} \mathrm{v}^{-1}\right)$ and the corresponding ketone (30 or 50 $\mathrm{mM}$ ). The reactions were shaken at $30^{\circ} \mathrm{C}$ and $250 \mathrm{rpm}$ for $24 \mathrm{~h}$ and stopped by extraction with ethyl acetate $(2 \times 0.5 \mathrm{~mL})$. The organic layer was separated by centrifugation ( $2 \mathrm{~min}, 13000 \mathrm{rpm})$ and dried over $\mathrm{Na}_{2} \mathrm{SO}_{4}$. Conversions and $e e$ of the corresponding alcohols were determined by GC (see Tables 1 and S1). For $\alpha$-brominated ketones, in order to avoid undesired $\mathrm{S}_{\mathrm{N}} 2$ reactions with Tris. $\mathrm{HCl}$ buffer, ${ }^{13}$ Tris. $\mathrm{H}_{2} \mathrm{SO}_{4}$ buffer $50 \mathrm{mM}$ pH $7.5(570 \mu \mathrm{L}, 1 \mathrm{mM}$ NADH) was used.

Enzymatic reduction using $E$. coli/ADH-A in the absence of the nicotinamide cofactor. In a 1.5 mL Eppendorf vial, E. coli/ADH-A (20 mg) was added in Tris-HCl buffer $50 \mathrm{mM} \mathrm{pH} 7.5(540 \mu \mathrm{L})$ and mixed with 2-propanol $\left(60 \mu \mathrm{L}, 10 \% \mathrm{v} \mathrm{v}^{-1}\right)$ and the corresponding ketone (100 or $\left.200 \mathrm{mM}\right)$. The reactions were shaken at $30^{\circ} \mathrm{C}$ and $250 \mathrm{rpm}$ for $24 \mathrm{~h}$ and stopped by extraction with ethyl acetate $(2 \times 0.5 \mathrm{~mL})$. The organic layer was separated by centrifugation (2 min, $13000 \mathrm{rpm}$ ) and dried over $\mathrm{Na}_{2} \mathrm{SO}_{4}$. Conversions and ee of the corresponding alcohols were determined by GC.

Enzymatic reduction of ketones at 100 or $200 \mathrm{mM}$ concentration using LBADH. In a $1.5 \mathrm{~mL}$ Eppendorf vial, LBADH (3 U) was added in Tris.HCl buffer $50 \mathrm{mM}$ pH $7.5\left(540 \mu \mathrm{L}, 1 \mathrm{mM} \mathrm{NADP}^{+}, 1\right.$ $\left.\mathrm{mM} \mathrm{MgCl}_{2}\right)$ and mixed with 2-propanol $\left(60 \mu \mathrm{L}, 10 \% \mathrm{v} \mathrm{v}^{-1}\right)$ and the corresponding ketone (100-200 $\mathrm{mM}$ ). The reactions were shaken at $30^{\circ} \mathrm{C}$ and $250 \mathrm{rpm}$ for $24 \mathrm{~h}$ and stopped by extraction with ethyl acetate $(2 \times 1 \mathrm{~mL})$. The organic layer was separated by centrifugation ( $2 \mathrm{~min}, 13000 \mathrm{rpm})$ and dried over $\mathrm{Na}_{2} \mathrm{SO}_{4}$. Conversions and $e e$ of the corresponding alcohols were determined by (chiral) GC (see Table $\mathrm{S} 1)$. For $\alpha$-brominated ketones, in order to avoid undesired $\mathrm{S}_{\mathrm{N}} 2$ reactions with Tris.HCl buffer, ${ }^{13}$ Tris. $\mathrm{H}_{2} \mathrm{SO}_{4}$ buffer $50 \mathrm{mM}$ pH $7.5(540 \mu \mathrm{L}, 1 \mathrm{mM} \mathrm{NADP}+, 1 \mathrm{mM} \mathrm{MgBr} 2)$ was used.

Enzymatic reduction of ketones at 100 or $200 \mathrm{mM}$ concentration using $E$. coli/ADH-A. In a 1.5 mL Eppendorf vial, E. coli/ADH-A (20 mg) was added in Tris.HCl buffer $50 \mathrm{mM}$ pH $7.5(540 \mu \mathrm{L}, 1$ 
mM NADH) and mixed with 2-propanol $\left(60 \mu \mathrm{L}, 10 \% \mathrm{v} \mathrm{v}^{-1}\right)$ and the corresponding ketone (100-200 $\mathrm{mM}$ ). The reactions were shaken at $30^{\circ} \mathrm{C}$ and $250 \mathrm{rpm}$ for $24 \mathrm{~h}$ and stopped by extraction with ethyl acetate $(2 \times 1 \mathrm{~mL})$. The organic layer was separated by centrifugation ( $2 \mathrm{~min}, 13000 \mathrm{rpm})$ and dried over $\mathrm{Na}_{2} \mathrm{SO}_{4}$. Conversions and $e e$ of the corresponding alcohols were determined by (chiral) GC (see Table $\mathrm{S} 1)$. For $\alpha$-brominated ketones, in order to avoid undesired $\mathrm{S}_{\mathrm{N}} 2$ reactions with Tris.HCl buffer, ${ }^{13}$ Tris. $\mathrm{H}_{2} \mathrm{SO}_{4}$ buffer $50 \mathrm{mM}$ pH $7.5(540 \mu \mathrm{L}, 1 \mathrm{mM}$ NADH) was used.

Enzymatic reduction of ketones at $0.5 \mathrm{M}$ concentration using LBADH. In a $1.5 \mathrm{~mL}$ Eppendorf vial, LBADH (3 U) was added in Tris. $\mathrm{HCl}$ buffer $50 \mathrm{mM}$ pH $7.5\left(480 \mu \mathrm{L}, 1 \mathrm{mM} \mathrm{NADP}{ }^{+}, 1 \mathrm{mM} \mathrm{MgCl}{ }_{2}\right)$ and mixed with 2-propanol $\left(120 \mu \mathrm{L}, 20 \% \mathrm{v} \mathrm{v}^{-1}\right)$ and the corresponding ketone $(0.5 \mathrm{M})$. The reaction was shaken at $30^{\circ} \mathrm{C}$ and $250 \mathrm{rpm}$ for $24 \mathrm{~h}$ and stopped by extraction with ethyl acetate $(2 \times 0.5 \mathrm{~mL})$. The organic layer was separated by centrifugation (2 min, $13000 \mathrm{rpm}$ ) and dried over $\mathrm{Na}_{2} \mathrm{SO}_{4}$. Conversions and $e e$ of the corresponding alcohols were determined by GC (see Figure 1 and Table S1). For $\alpha$ brominated ketones, in order to avoid undesired $\mathrm{S}_{\mathrm{N}} 2$ reactions with Tris. $\mathrm{HCl}$ buffer, ${ }^{13} \mathrm{Tris}^{\mathrm{H}} \mathrm{H}_{2} \mathrm{SO}_{4}$ buffer $50 \mathrm{mM}$ pH $7.5\left(480 \mu \mathrm{L}, 1 \mathrm{mM} \mathrm{NADP}^{+}, 1 \mathrm{mM} \mathrm{MgBr} 2\right)$ was used.

Scale-up of the bioreductions using LBADH. In a $10 \mathrm{~mL}$ glass vial, LBADH (15 U) was added in Tris. $\mathrm{HCl}$ buffer $50 \mathrm{mM}$ pH $7.5\left(1 \mathrm{mM} \mathrm{NADP}^{+}, 1 \mathrm{mM} \mathrm{MgCl}_{2}\right)$ and mixed with 2-propanol $\left(10 \% \mathrm{v} \mathrm{v}^{-1}\right)$ and the corresponding ketone $(50 \mathrm{mg}, 100 \mathrm{mM})$. The reaction was shaken at $30^{\circ} \mathrm{C}$ and $250 \mathrm{rpm}$ for $24 \mathrm{~h}$ and stopped by extraction with diethyl ether $(2 \times 5 \mathrm{~mL})$. The organic layer was dried over $\mathrm{Na}_{2} \mathrm{SO}_{4}$ and the solvent was carefully evaporated (CAUTION: fluorohydrins are highly volatile). Conversions and $e e$ of the corresponding alcohols were determined by GC and NMR. For $2 \mathbf{i}$, in order to avoid undesired $\mathrm{S}_{\mathrm{N}} 2$ reactions with Tris. $\mathrm{HCl}$ buffer, ${ }^{13}$ Tris. $\mathrm{H}_{2} \mathrm{SO}_{4}$ buffer $50 \mathrm{mM}$ pH 7.5 (1 mM NADP $\left.{ }^{+}, 1 \mathrm{mM} \mathrm{MgBr}_{2}\right)$ was used. In most cases, the final enantiopure products exhibited excellent purity after solvent evaporation, so no further purification was necessary. To obtain $(S)-\mathbf{6 i},(S)-\mathbf{7 f}$ and $(S)-\mathbf{7 j}$, flash chromatography was employed to purify the alcohol derivatives. $(S)-6 \mathbf{i}(37 \mathrm{mg}, 73 \%$ yield, >99\% ee $),(S)-7 \mathbf{a}(47 \mathrm{mg}, 93 \%$ yield, >99\% ee), $(S)-7 \mathbf{b}(44 \mathrm{mg}, 87 \%$ yield, >99\% ee), $(S)-7 \mathbf{c}(36 \mathrm{mg}, 71 \%$ yield, >99\% ee $),(S)-7 \mathbf{d}(46$ mg, 91\% yield, >99\% ee), (S)-7e (44 mg, 87\% yield, >99\% ee), $(S)-7 f(33 \mathrm{mg}, 64 \%$ yield, >99\% ee $)$, 
$(S)-7$ g (48 mg, 94\% yield, >99\% ee), $(S)-7$ h (48 mg, 95\% yield, >99\% ee), $(S)-7 \mathbf{i}(42 \mathrm{mg}, 83 \%$ yield, >99\% ee), (S)-7j (36 mg, 71\% yield, >99\% ee), $(S)-7 \mathbf{k}(41 \mathrm{mg}, 81 \%$ yield, >99\% ee), $(S)-7 \mathbf{l}(45 \mathrm{mg}$, 88\% yield, >99\% ee), $(S)-8$ (42 mg, 83\% yield, >99\% ee), and $(S)-9(43 \mathrm{mg}, 85 \%$ yield, >99\% ee).

Scale-up of the bioreduction of ketone 2 b employing $\boldsymbol{E}$. coli/ADH-A. In a $25 \mathrm{~mL}$ Erlenmeyer flask, E. coli/ADH-A (460 mg) was added in Tris. $\mathrm{H}_{2} \mathrm{SO}_{4}$ buffer $50 \mathrm{mM}$ pH $7.5(10.8 \mathrm{~mL}, 1 \mathrm{mM} \mathrm{NADH})$ and mixed with 2-propanol (1.2 mL, 10\% $\left.\mathrm{v} \mathrm{v}^{-1}\right)$ and ketone $\mathbf{2 b}(260 \mathrm{mg}, 100 \mathrm{mM})$. Reactions were shaken at $30^{\circ} \mathrm{C}$ and $250 \mathrm{rpm}$ for $24 \mathrm{~h}$. After that time the pellet was separated by centrifugation (15 min, 6000 $\mathrm{rpm})$ and extracted with ethyl acetate $(3 \times 20 \mathrm{~mL})$. The organic layers were combined and dried over $\mathrm{Na}_{2} \mathrm{SO}_{4}$. The solvent was evaporated and the crude was purified by flash chromatography. Isolated yield of $(R)-6 \mathbf{b}: 184 \mathrm{mg}, 70 \%,>99 \%$ ee.

2-Bromo-1-(4-fluorophenyl)ethanol (6b). ${ }^{\mathbf{2 3}} \mathrm{H} \mathrm{NMR}^{1}\left(300 \mathrm{MHz}, \mathrm{CDCl}_{3}\right): \delta 7.39$ (m, 2H), $7.08($ ap t, $J=8.7 \mathrm{~Hz}, 2 \mathrm{H}), 4.93(\mathrm{~m}, 1 \mathrm{H}), 3.65-3.50(\mathrm{~m}, 2 \mathrm{H}), 2.72(\mathrm{~d}, J=3.2 \mathrm{~Hz}) ;{ }^{13} \mathrm{C} \mathrm{NMR}\left(75 \mathrm{MHz}, \mathrm{CDCl}_{3}\right): \delta$ $162.6(\mathrm{~d}, J=245.3 \mathrm{~Hz}), 136.0(\mathrm{~d}, J=3.0 \mathrm{~Hz}), 127.7(\mathrm{~d}, J=8.3 \mathrm{~Hz}), 115.6(\mathrm{~d}, J=21.4 \mathrm{~Hz}), 73.2,40.1$; ${ }^{19} \mathrm{~F} \mathrm{NMR}\left(282 \mathrm{MHz}, \mathrm{CDCl}_{3}\right): \delta-113.4$.

Acknowledgments. W.B. thanks the Principado de Asturias for her predoctoral fellowship (Severo Ochoa Program). I.L. thanks the Spanish Ministerio de Ciencia e Innovación (MICINN) for personal funding (Ramón y Cajal Program). Financial support of this work by the Spanish MICINN (Project MICINN-12-CTQ2011-24237) is gratefully acknowledged.

Supporting Information Available. Enzymatic conversions and ee at higher substrate concentrations (Table S1), analytical methods, and copies of ${ }^{1} \mathrm{H}-,{ }^{13} \mathrm{C}-$, and ${ }^{19} \mathrm{~F}-\mathrm{NMR}$ for new compounds are described. This material is available free of charge via the Internet at http://pubs.acs.org. 


\section{References}

1. (a) Purser, S.; Moore, P. R.; Swallow, S.; Gouverneur, V. Chem. Soc. Rev. 2008, 37, 320-330. (b) Ametamey, S. M.; Honer, M.; Schubiger, P. A. Chem. Rev. 2008, 108, 1501-1516.

2. Kusumoto, T.; Hiyama, T. in Enantiocontrolled Synthesis of Fluoro-Organic Compounds: Stereochemical Challenges and Biomedicinal Targets, Soloshonok, V. A., Ed.; Wiley: Chichester, 1999, pp. 535-556.

3. For some recent examples, see: (a) Cresswell, A. J.; Davies, S. G.; Lee, J. A.; Morris, M. J.; Roberts, P. M.; Thomson, J. E. J. Org. Chem. 2012, 77, 7262-7281. (b) Chen, J.-L.; Zheng, F.; Huang, Y.-E.; Qing, F.-L. J. Org. Chem. 2011, 76, 6525-6533.

4. (a) Hollingworth, C.; Gouverneur, V. Chem. Commun. 2012, 48, 2929-2942. (b) Furuya, T.; Kamlet, A. S.; Ritter, T. Nature 2011, 473, 470-477. (c) Haufe, G. J. Fluor. Chem. 2004, 125, 875-894.

5. Kalow, J. A.; Doyle, A. G. J. Am. Chem. Soc. 2011, 133, 16001-16012.

6. Arroyo, Y.; Sanz-Tejedor, M. A.; Parra, A.; García Ruano, J. L. Chem. Eur. J. 2012, 18, 5314 5318.

7. Mohanta, P. K.; Davis, T. A.; Gooch, J. R.; Flowers, R. A., II J. Am. Chem. Soc. 2005, 127, 1189611897.

8. (a) Enzyme Catalysis in Organic Synthesis, 3rd ed.; Drauz, K., Gröger, H., May, O., Eds.; WileyVCH: Weinheim, 2012. (b) Faber, K. Biotransformations in Organic Chemistry, 6th ed.; Springer: Berlin, 2011.

9. Some selected examples: (a) Barkakaty, B.; Takaguchi, Y.; Tsuboi, S. Tetrahedron 2007, 63, 970976. (b) Nakamura, K.; Yamanaka, R. Tetrahedron: Asymmetry 2002, 13, 2529-2533. (c) Matsuda, T.; Harada, T.; Nakajima, N.; Itoh, T.; Nakamura, K. J. Org. Chem. 2000, 65, 157-163. 
10. Recent bibliography: (a) Hollmann, F.; Arends, I. W. C. E.; Holtmann, D. Green Chem. 2011, 13, 2285-2313. (b) García-Urdiales, E.; Alfonso, I.; Gotor, V. Chem. Rev. 2011, 111, PR110-PR180. (c) Musa, M. M.; Phillips, R. S. Catal. Sci. Technol. 2011, 1, 1311-1323.

11. (a) Xu, G.-C.; Yu, H.-L.; Zhang, X.-Y.; Xu, J.-H. ACS Catal. 2012, 2, 2566-2571. (b) Itoh, N.; Isotani, K.; Nakamura, M.; Inoue, K.; Isogai, Y.; Makino, Y. Appl. Microbiol. Biotechnol. 2012, 93, 1075-1085. (c) Wang, L.-J.; Li, C.-X.; Ni, Y.; Zhang, J.; Liu, X.; Xu, J.-H. Bioresour. Technol. 2011, 102, 7023-7028. (d) Grau, B. T.; Devine, P. N.; DiMichele, L. N.; Kosjek, B. Org. Lett. 2007, 9, 49514954.

12. Schirlin, D.; Baltzer, S.; VanDorsselaer, V.; Weber, F.; Weill, C.; Altenburger, J. M.; Neises, B.; Flynn, G.; Rimy, J. M.; Tarnus, C. Bioorg. Med. Chem. Lett. 1993, 3, 253-258.

13. Bisogno, F. R.; Cuetos, A.; Orden, A. A.; Kurina-Sanz, M.; Lavandera, I.; Gotor, V. Adv. Synth. Catal. 2010, 352, 1657-1661.

14. Adapted from: Zizhan, C.; Zhu, W.; Zheng, Z.; Zou, X. J. Fluor. Chem. 2010, 131, 340-344.

15. Mąkosza, M.; Bujok, R. J. Fluor. Chem. 2005, 126, 209-216.

16. (a) Cuetos, A.; Bisogno, F. R.; Lavandera, I.; Gotor, V. Chem. Commun. 2013, 49, 2625-2627. (b) Cuetos, A.; Rioz-Martínez, A.; Bisogno, F. R.; Grischek, B.; Lavandera, I.; de Gonzalo, G.; Kroutil, W.; Gotor, V. Adv. Synth. Catal. 2012, 354, 1743-1749. (c) Kurina-Sanz, M.; Bisogno, F. R.; Lavandera, I.; Orden, A. A.; Gotor, V. Adv. Synth. Catal. 2009, 351, 1842-1848.

17. This $(S)$-selective enzyme has a strong preference for NADH/NAD ${ }^{+}$. See: Edegger, K.; Gruber, C. C.; Poessl, T. M.; Wallner, S. R.; Lavandera, I.; Faber, K.; Niehaus, F.; Eck, J.; Oehrlein, R.; Hafner, A.; Kroutil, W. Chem. Commun. 2006, 2402-2404.

18. This $(R)$-selective enzyme shows a preference for NADPH/NADP ${ }^{+}$. See, for instance: Wolberg, M.; Hummel, W.; Wandrey, C.; Müller, M. Angew. Chem., Int. Ed. 2000, 39, 4306-4308. 
19. (a) Ma, H.; Yang, L.; Ni, Y.; Zhang, J.; Li, C.-X.; Zheng, G.-W.; Yang, H.; Xu, J.-H. Adv. Synth. Catal. 2012, 354, 1765-1772. (b) Shen, N.-D.; Ni, Y.; Ma, H.-M.; Wang, L.-J.; Li, C.-X.; Zheng, G.W.; Zhang, J.; Xu, J.-H. Org. Lett. 2012, 14, 1982-1985.

20. Bisogno, F. R.; García-Urdiales, E.; Valdés, H.; Lavandera, I.; Kroutil, W.; Suárez, D.; Gotor, V. Chem. Eur. J. 2010, 16, 11012-11019.

21. Burnett, D. A. Curr. Med. Chem. 2004, 11, 1873-1887.

22. Karlsson, S.; Sörensen, J. H. Org. Process Res. Dev. 2012, 16, 586-594.

23. Ren, J.; Dong, W.; Yu, B.; Wu, Q.; Zhu, D. Tetrahedron: Asymmetry 2012, 23, 497-500.

24. (a) Macharla, A. K.; Nappunni, R. C.; Marri, M. R.; Peraka, S.; Nama, N. Tetrahedron Lett. 2012, 53, 191-195. (b) Duca, M.; Malnuit, V.; Barbault, F.; Benhida, R. Chem. Commun. 2010, 46, 61626164. (c) Richards, J. J.; Melander, C. J. Org. Chem. 2008, 73, 5191-5193. (d) Pravst, I.; Zupan, M.; Stavber, S. Tetrahedron 2008, 64, 5191-5199.

25. Raja, E. K.; Klumpp, D. A. Tetrahedron Lett. 2011, 52, 5170-5172.

26. (a) Castro, L. C. M.; Bezier, D.; Sortais, J.-B.; Darcel, C. Adv. Synth. Catal. 2011, 353, 12791284. (b) Beier, P.; Alexandrova, A. V.; Zibinsky, M.; Surya Prakash, G. K. Tetrahedron 2008, 64, 10977-10985. (c) Fuglseth, E.; Sundby, E.; Bruheim, P.; Hoff, B. H. Tetrahedron: Asymmetry 2008, 19, 1941-1946. (d) Liu, J.; Ni, C.; Li, Y.; Zhang, L.; Wang, G.; Hu, J. Tetrahedron Lett. 2006, 47, 67536756. (e) Stavber, G.; Zupan, M.; Jereb, M.; Stavber, S. Org. Lett. 2004, 6, 4973-4976. (f) Sibille, S.; Mcharek, S.; Perichon, J. Tetrahedron 1989, 45, 1423-1428.

27. It was not possible to measure the MS spectra of this compound since it decomposed in all conditions tried. 